\title{
Medium-term Culture of Normal Human Oral Mucosa: A Novel Three-dimensional Model to Study the Effectiveness of Drugs Administration
}

\author{
Fabio Bucchieri ${ }^{1,5, *}$, Alberto Fucarino ${ }^{1}$, Antonella Marino Gammazza ${ }^{1,5}$, Alessandro Pitruzzella ${ }^{1,5}$, Vito \\ Marcianò ${ }^{1}$, Carlo Paderni ${ }^{3}$, Viviana De Caro ${ }^{2}$, Maria Gabriella Siragusa ${ }^{2}$, Lorenzo Lo Muzio ${ }^{6}$, Stephen T \\ Holgate $^{4}$, Donna E Davies ${ }^{4}$, Felicia Farina ${ }^{1}$, Giovanni Zummo ${ }^{1}$, Yasusei Kudo ${ }^{7}$, Italo Libero Giannola ${ }^{2}$ and \\ Giuseppina Campisi ${ }^{3}$
}

\begin{abstract}
${ }^{1}$ Dpt di Biomedicina Sperimentale e Neuroscienze Cliniche Università di Palermo, Palermo, Italy; ${ }^{2}$ Dpt di Scienze e Tecnologie Molecolari e Biomolecolari, Università di Palermo, Palermo, Italy; ${ }^{3}$ Dpt di Discipline Chirurgiche ed Oncologiche, Università di Palermo, Palermo, Italy; ${ }^{4}$ Clinical and Experimental Sciences, University of Southampton, Faculty of Medicine, Sir Henry Wellcome Laboratories, Southampton General Hospital, Southampton, UK; ${ }^{5}$ Istituto Euro-Mediterraneo di Scienza e Tecnologia, IEMEST, Palermo, Italy; ${ }^{6}$ Dpt. di Scienze Chirurgiche, Università di Foggia, Italy; ${ }^{7}$ Dpt. Oral and Maxillofacial Pathobiology, Division of Frontier Medical Science, Graduate School of Biomedical Sciences, Hiroshima University, Japan
\end{abstract}

\begin{abstract}
Tissue-engineered oral mucosal equivalents have been developed for in vitro studies for a few years now. However, the usefulness of currently available models is still limited by many factors, mainly the lack of a physiological extracellular matrix (ECM) and the use of cell populations that do not reflect the properly differentiated cytotypes of the mucosa of the oral cavity. For this reason, we have developed a novel three-dimensional culture model reflecting the normal architecture of the human oral mucosa, with the main aim of creating a better in vitro model where to test cellular responses to drugs administration. This novel 3D cell culture model (3D outgrowth) was set up using an artificial extracellular matrix (Matrigel ${ }^{\mathrm{TM}}$ ), allowing the interactions required for proper differentiation of the various citotypes which form the mucosal layer. Biopsies of human oral mucosa, in fragments of about $0.5 \mathrm{~mm}^{3}$, were placed onto $6.5 \mathrm{~mm}$ Transwells, covered with Matrige ${ }^{\mathrm{TM}}$ and grown in a specific culture medium. A gradual formation of an architectural structure similar to that of the in vivo oral mucosa was observed. Transmission electron and confocal microscopy were employed to characterize the newly developed model: the cell components (keratinocytes and fibroblasts) differentiated properly within the outgrowth and reconstituted, in vitro, the physiological structure of the human oral mucosa, including a stratified non-keratinized squamous layer composed of four different layers, a proper basal membrane and a lamina propria where fibroblasts produce ECM. Moreover, keratinocytes expressed CK5, CK13, CK19 and E-cadherin, whereas fibroblasts expressed collagen type I and IV, laminin and fibronectin.
\end{abstract}

3D outgrowths could be considered a valid alternative to animal models, and provide useful information for researchers interested in studying the responses of the human oral mucosa to locally delivered drugs or other exogenous treatments.

Keywords: Tissue-engineering, human oral mucosa, 3D outgrowth model, keratinocytes, fibroblasts, transmission electron microscopy, immunofluorescence, immunogold.

\section{INTRODUCTION}

Normal cells in the human body exist in a three-dimensional environment, completely surrounded by other cells, membranes, fibrous layers and adhesion proteins. But culturing cells on flat plastic flasks or plates results in artificial two-dimensional sheets of cells. Three-dimensional (3D) cultures mimic the in vivo cell environment providing more physiologically significant information compared to in vitro cell culture models; however, there are several variables inherently associated with these assays that can influence the success of $3 \mathrm{D}$ cultures such as matrix composition, cell type, cell health, cell seeding densities, time of culture and basement membrane extract. The situation is further complicated when the creation of a 3D tissue equivalent containing different types of cells organized in a complex structure is attempted, entering in the experimental field of tissue engineering.

Tissue engineering has progressed greatly in the last twenty years or so, especially with regard to recreating function of specific tissues such as exocrine pancreas, cartilage, bone, skin and blood vessels. However, proper design models still need to be developed for engineering complex 3D ECM microenvironments to understand disease progression. In fact, the main objectives of modern tissue engineering comprise repeating specific tissue functions for

*Address correspondence to this author at the Dpt di Biomedicina Sperimentale e Neuroscienze Cliniche Università di Palermo, Palermo, Italy; E-mail: fabio.bucchieri@unipa.it regenerative medicine and developing in vitro models of human tissues to investigate disease pathogenesis, as well as for testing and screening new medications before expensive clinical trials. For these objectives to be successfully achieved, it is fundamental for the engineered models to recreate, in vitro, the complex in vivo interactions between cells and their microenvironments.

Tissue-engineered oral mucosal equivalents have been developed for in vitro biocompatibility studies, as well as for mucosal irritation and oral disease studies with the aim to better understand disease process and discover new treatments $[1,2]$. In the last decade, research has concentrated on the characterization of human mucosal equivalent by introducing new dermal scaffold and epithelial cell culture methods. In 1975, Rheinwald and Green introduced a method to grow human keratinocytes in in vitro serial cultures, using a feeder layer composed of irradiated mouse fibroblasts and a specific culture medium [3]. This method is widely used for the culture of keratinocytes and single-layer epithelial sheets, but such sheets are fragile, difficult to handle and tend to contract [1]. Multilayer sheets of cultured epithelium were obtained by culturing oral keratinocytes and fibroblasts, crucial for the production of extracellular matrix, on permeable ethylene terephthalate cell culture membrane (PET) at the air/liquid interface [4] or in polycarbonate cell culture inserts, developed by SkinEthic Laboratories (Nice, France). These models have the characteristic of being very similar to native epithelium and showing signs of differentiation, such as different cytokeratin expression and basement membrane formation, but not 
to the point of full differentiation because the cells used are derived from oral squamous carcinoma [1]. Moreover, the scaffold that supports the cells is an important element in oral mucosa reconstruction. The currently available scaffolds can be divided into several categories: naturally derived scaffolds, such as acellular dermis and amniotic membrane [5]; fibroblast-populated skin substitutes [6]; pure collagen scaffolds and collagen-based matrices [7]; gelatin-based scaffolds such as gelatin-hyaluronate and gelatinchitosan-hyaluronic acid; fibrin-based materials; synthetic scaffolds, such as elastin-like recombinant polymer [2] and hybrid scaffolds, which are combinations of natural and synthetic matrices [1]. The benefit of pure or collagen-based matrices is that the collagen gel supports fibroblasts, which provides a suitable substrate for keratinocyte multilayer formation, but it also biodegrades rapidly and has poor mechanical properties. However, the fibroblasts embedded in the collagen gel have been shown to synthesize less ECM than those cultured on three-dimensional porous scaffolds. Potential weaknesses of artificial scaffolds include lack of porosity, poor fibroblast infiltration, and contraction of the scaffold when large numbers of fibroblasts are seeded [4]. Moreover, the incorporation of fibroblast-populated collagen into the pore of the scaffold is required to obtain better results [8]. Taking into account the notions discussed above, we have recently focused our research efforts on tissue-engineering a novel 3D model of the normal human oral mucosa, to overcome some of the shortcomings of the current in vitro models. Our model includes two cell populations (keratinocytes and fibroblasts) that outgrow from an oral biopsy fragment into a natural extracellular 3D matrix (Matrigel ${ }^{\mathrm{TM}}$ ) that initially drives the outgrowth of the cells, and is completely replaced during the culture period by a newly deposed matrix produced by the fibroblasts. Matrigel $^{\mathrm{TM}}$ is a gelatinous protein mixture secreted by Engelbreth-Holm-Swarm (EHS) mouse sarcoma cells. This mixture resembles the complex extracellular environment found in many tissues and is considered to be a good cell culture substrate for its heterogeneous composition. The major components of Matrigel ${ }^{\mathrm{TM}}$ are basement membrane proteins such as laminin, entactin and collagen IV, which present cultured cells with the adhesive peptide sequences that they would encounter in their natural environment [19]. Matrigel ${ }^{\mathrm{TM}}$ also contains growth factors that promote differentiation and proliferation of many cell types. Moreover, Matrigel $^{\mathrm{TM}}$ constitutes a uniform and controllable structure, very important for accuracy and reproducibility of an in vitro model for compound testing use [4].

Our hypothesis was that this kind of model could exhibit a proper histological architecture and biochemical composition (two essential features required of an in vitro model that is to be employed to study the responses to exogenous modifications of its microenvironment, such as those that take place during drug administration assays). In order to achieve this goal, we characterized the outgrowth model both at an ultra-structural and at an immunophenotypic level.

\section{MATERIALS AND METHODS}

\subsection{Materials}

\subsubsection{Chemicals}

$\mathrm{HCl}$ was purchased from Titolchimica (Pontecchio, Italy) and isopropanol from Carlo Erba (Milan, Italy). Glutaraldehyde, osmium tetroxide $\left(\mathrm{OsO}_{4}\right)$, epoxy resin (Epon812) and epoxy accelerator (DMP30) were purchased from Electron Microscopy Sciences (Hatfield, PA, USA). 1,2-Propylene oxide, $\mathrm{NaH}_{2} \mathrm{PO}_{4} * 2 \mathrm{H}_{2} \mathrm{O}$, $\mathrm{NaC}_{6} \mathrm{H}_{5} \mathrm{O}_{7} * \mathrm{H}_{2} \mathrm{O}$ and $\mathrm{NaOH}$ were purchased from Merck (Darmstadt, Germany). $\mathrm{UO}_{2}\left(\mathrm{CH}_{3} \mathrm{COO}\right) 2 \cdot 2 \mathrm{H}_{2} \mathrm{O}$ and $\mathrm{Pb}\left(\mathrm{NO}_{3}\right)_{2}$ were purchased from Sigma-Aldrich (Milan, Italy).

All chemicals and solvents were of analytical grade and were used without further purification.

\subsubsection{Cell Culture Reagents}

Recombinant human EGF (hEGF) was purchased from Cell Signaling Technology (Beverly, MA, USA). L-Glutamine was obtained from Stem Cell Technologies (Tukwila, WA, USA). Dulbecco's Modified Eagle Medium (DMEM) was purchased from Sigma-Aldrich (Milan, Italy). GIBCO ${ }^{\text {TM }}$ Keratinocyte-Serum Free Medium (Keratinocyte-SFM) was obtained from Invitrogen life technologies (Carlsbad, CA, USA). Fetal bovine Serum (FBS), Hanks BSS without phenol red (HBSS) and Phosphate Buffered Solution (PBS) were purchased from Lonza (Cologne, Germany).

\subsection{Methods}

\subsubsection{Three-dimensional Oral Outgrowth Model}

Human oral mucosa biopsies were obtained from patients referred to the Unit of Oral Medicine of the University of Palermo. The following procedures were adopted, conforming to the relevant ethical guidelines for human research and in agreement with the Helsinki Declaration of 1975 as revised in 1983, as well as approved by the Ethic Council of the Polyclinic of the University of Palermo, Italy. Written informed consents were obtained from all patients. Each patient was subjected to topical antifungal therapy (miconazole 2\% oral gel, Daktarin, Janssen-Cilag) 3 times/day for seven days before the biopsy. After a 1 minute oral rinse with $0.2 \%$ chlorhexidine, oral mucosa samples were obtained by a $6 \mathrm{~mm} \mathrm{di-}$ ameter biopsy punch on the margin of the lesion with clinically healthy tissue. Each sample was divided into two parts. The outer part of the sample was fixed in formalin and sent for histocitopathological examination, while the inner part was immediately placed in fresh culture medium and processed for the 3D oral outgrowths. A total of six subjects, whose histo-citopathological examination resulted negative for dysplastic/cancerous lesions of the oral cavity, were recruited for this study.

Biopsies were washed several times in PBS, subsequently cut into $0.5 \mathrm{~mm}^{3}$ pieces using a sterile scalpel, and placed in the middle of $6.5 \mathrm{~mm}$ Transwells on a nylon membrane (Becton Dickinson,


(Becton Dickinson). The Transwells were placed on 24 well culture plates (Corning Life Sciences), and these were then kept at $37^{\circ} \mathrm{C}$ for 5 minutes to facilitate Matrigel ${ }^{\mathrm{TM}}$ jellification. $330 \mu \mathrm{l}$ of growth medium mix was then added to each well. This mix was constituted of Keratinocyte-SFM supplemented with $5 \mathrm{ng} / \mathrm{ml}$ of h-EGF and DMEM supplemented with $10 \%$ FBS (1: 1), which was placed underneath the nylon membrane of the Transwells. The outgrowths were cultured at $37^{\circ} \mathrm{C}$ in a $5 \% \mathrm{CO} 2$ atmosphere and the medium was changed every 48 hours. An inverted light microscope equipped with phase contrast rings (LEICA DM-IRB, Leica Microsystems Srl, Milan, Italy) was used to monitor the outgrowths. After 15 days in culture the outgrowths were prepared for electron microscopy and immunostaining as described below. By this time point, the has been completely dissolved by the proteolytic activity of the cells and therefore there is no need to separate the outgrowths from the Matrigel ${ }^{\mathrm{TM}}$.

\subsubsection{Electron Microscopy}

Immediately after excision of the nylon membrane from the Transwells using a scalpel, the outgrowths were fixed in a $2.5 \%$ glutaraldehyde solution in phosphate buffer, $\mathrm{pH} 7.4$, for 20 minutes at room temperature. Phosphate-buffered saline (PBS) $\mathrm{Ca}^{2+}$ and $\mathrm{Mg}^{2+-}$ free solution ( $\mathrm{pH}$ 7.4) was prepared by dissolving $\mathrm{KH}_{2} \mathrm{PO}_{4}$ $(0.144 \mathrm{~g})$, anhydrous $\mathrm{Na}_{2} \mathrm{HPO}_{4}(0.795 \mathrm{~g})$ and $\mathrm{NaCl}(9.0 \mathrm{~g})$ in 1 litre of distilled water. After fixation, the glutaraldehyde was removed and the outgrowths were stored in Millonig's Buffer. 25,6g of $\mathrm{NaH}_{2} \mathrm{PO}_{4} \times 2 \mathrm{H}_{2} \mathrm{O}$ was added to 1 litre of distilled water to make the A solution of Millonig's Buffer. To obtain the B solution, 25,2g of $\mathrm{NaOH}$ was added in 1 litre of distilled water. Finally, $83 \mathrm{ml}$ of the A solution was mixed with $17 \mathrm{ml}$ of the B solution to make $100 \mathrm{ml}$ of Buffer; the $\mathrm{pH}$ was adjusted to 7.4. After 3 consecutive washes 
in Millonig's Buffer, the pieces were post-fixed in $1 \% \mathrm{OsO}_{4}$ for $2 \mathrm{~h}$, dehydrated in an ascending ethanol series, treated for 30' in propylene oxide, infiltrated with epoxy resin (Epon812, Electron Microscopy Science, Hatfield, PA, USA) in propylene oxide (1:3, 1:2, 1: 1 for 30 minutes at room temperature respectively), and finally embedded in Epon812 with DMP30. The resin was then polymerized at $60^{\circ} \mathrm{C}$ for 48 hours. Ultrathin and semithin sections were cut with an ultramicrotome (Ultracut E, Reichert-Jung, Depew, NY, USA) at different thicknesses and mounted on copper and gold grids or on glass slides for further use. Contrast solution for the grids to be used for electron microscopy was prepared by dissolving $0,7 \mathrm{~g}$ of uranyl acetate in $10 \mathrm{ml}$ of methanol; Reynolds' solution was prepared by dissolving $1,33 \mathrm{~g}$ of $\mathrm{Pb}\left(\mathrm{NO}_{3}\right)_{2}$ and $1,76 \mathrm{~g} \mathrm{NaC} \mathrm{N}_{6} \mathrm{H}_{5} \mathrm{O}_{7} \mathrm{x}$ $\mathrm{H}_{2} \mathrm{O}$ and $8 \mathrm{ml}$ of $\mathrm{NaOH} 1 \mathrm{~N}$ in $50 \mathrm{ml}$ of distilled water at $\mathrm{pH} 12$.

\subsubsection{Indirect Immunofluorescence}

3D outgrowths were stained in situ at the appropriate time points. Briefly, outgrowths were washed once with $1 \mathrm{ml} /$ well of HBSS and fixed in situ in $500 \mu 1 /$ well of ice-cold absolute methanol for 20 minutes at $-20^{\circ} \mathrm{C}$. Outgrowths, inside their plastic supports, were then left to dry in a laminar flow cabinet for 30 minutes and stored at $-20^{\circ} \mathrm{C}$. Trays were defrosted at room temperature, and washed twice with $1 \mathrm{ml} /$ well of phosphate buffered saline (PBS), permeabilized with $500 \mu \mathrm{l} /$ well of Triton X-100 (Sigma, UK) $0.1 \%$ in PBS for 3 minutes on ice and washed once with $1 \mathrm{ml} /$ well of PBS. Unspecific binding sites were blocked with $250 \mu 1 /$ well of DMEM 10\% FBS for 15 minutes. During this period, the primary antibodies were diluted in incubation buffer (DMEM 10\%, Tween$200.1 \%$ and Sodium Azide 0.1\% in PBS). The blocking buffer was then removed, and without washing, the diluted antibodies were added to the wells for 45 minutes. Wells were then washed twice with $1 \mathrm{ml} /$ well of incubation buffer and, when needed, secondary antibodies were diluted in incubation buffer and applied to the wells for 45 minutes. Secondary antibodies were conjugated with different fluorochromes.

Table 1 shows a list of all the primary antibodies employed and their working dilutions.

The secondary antibodies were: a secondary Alexa Fluor647conjugated goat anti-rabbit Ab (1: 500; Molecular Probes, USA); a secondary FITC-conjugated goat anti-mouse IgG Ab (working dilution 1: 400, purchased from Sigma, UK); a secondary TRITCconjugated goat anti-mouse IgG Ab (working dilution 1: 500, purchased from Sigma, UK). At the end of the 45 minutes incubation with the secondary antibodies, wells were washed twice with $1 \mathrm{ml}$ of PBS and coverslips mounted with MOVIOL ${ }^{\circledR}(\mathrm{DABCO})$ mounting medium. Appropriate negative controls were prepared by replacing primary antibodies with proper isotype control sera.

The trays were then ready to be observed with a LEICA SP5 inverted confocal microscope (Leica, Heidelberg, Germany) with filters for FITC (excitation $488 \mathrm{~nm}$, emission 500-535 nm), TRITC (excitation $557 \mathrm{~nm}$, emission 560-600 nm), and Alexa Fluor647 (excitation $633 \mathrm{~nm}$, emission 640-680 nm). Each image was averaged from 14 scans within a thickness of 5 to $7 \mu \mathrm{m}$.

Table 1. List of primary antibodies used for immunostaining with their supplier, clone name, source and final dilution. In brackets is reported the dilution that was used for Immunogold.

\begin{tabular}{|c|c|c|c|c|}
\hline & Supplier & Clone & Source & Dilution \\
\hline CK10 & “ & AE20 & Mouse IgG1 & $1: 50$ \\
\hline CK13 & Abnova, USA & $1 \mathrm{C} 7$ & Mouse IgG2a & $1: 200$ \\
\hline CK20 & “ & E-9 & Mouse IgG1 & 1: 100 \\
\hline Collagen type I & Millipore, UK & 5D8-G9 & Mouse IgG1 & $1: 50$ \\
\hline Collagen type IV & Santa Cruz, UK & Col-94 & Mouse IgG1 & $1: 200$ \\
\hline Laminin & Millipore, UK & 2G6/A2 & Mouse IgG1 & $1: 200$ \\
\hline Involucrin & “ & $\mathrm{m}-116$ & Rabbit IgG & $1: 400$ \\
\hline MITF & “ & H-50 & Rabbit IgG & $1: 500$ \\
\hline CD3 & R\&D Systems, USA & UCHT1 & Mouse IgG1 & 1: 100 \\
\hline CD4 & “ & 34930 & Mouse IgG1 & $1: 50$ \\
\hline CD8 & “ & 37006 & Mouse IgG2b & $1: 50$ \\
\hline CD45 & “ & 2D1 & Mouse IgG1 & 1: 100 \\
\hline CD68 & “ & 298807 & Mouse IgG2b & $1: 100$ \\
\hline
\end{tabular}


Immunofluorescent staining was semi-quantified by scoring the percentage of positive cells or area (depending on the antigen of interest), evaluated by three different operators (AMG, AF and AP) in five different mid-magnification (400x) microscopic fields (score: - absence of immunostaining, $-/+$ less than $10 \%$ of the total number of cells/area scored positively; + between $10 \%$ and $50 \%$ scored positively; $++50-100 \%$ of the total number of cells/area were positive).

\subsubsection{Immunogold}

Ultrathin sections were mounted on gold grids to prepare them for the immunogold assay. The outgrowths were included in epoxy resin that notoriously covers antigenic sites, making the execution of immunological investigation techniques considerably more difficult. A pre-treatment to unmask the sites with sodium citrate was performed to ensure better results. Gold grids were placed in a baker filled with a sodium citrate solution and subsequently microwaved for 4 minutes at $850 \mathrm{~W}$.

The grids were then washed twice in PBS, and subsequently incubated in a serum blocking solution for 30 minutes and, without washing, the incubation proceeded with primary antibodies diluted in dilution buffer for 1 hour and half at room temperature. Primary antibodies used were against laminin, fibronectin and collagen type
IV and their dilutions can be found in Table 1. Appropriate negative controls were prepared by replacing primary antibodies with proper isotype control sera.Grids were rinsed five times with PBS for 3 min and incubated with secondary antibody conjugated with $10 \mathrm{~nm}$ colloidal gold particles for 30 minutes at room temperature, and washed again for five times with PBS for $3 \mathrm{~min}$. The grids were then fixed in a $2.5 \%$ glutaraldehyde solution in PBS for 15 minutes and finally washed five times in distilled water for 3 minutes. The grids were then prepared for contrast staining by treating them with uranyl acetate for 5 minutes, followed by eight washes with methanol for 2 minutes, treated with Reynolds' solution for 5 minutes and finally rinsed eight times in distilled water for 2 minutes. After this procedure, the grids were ready for electron microscopy.

\section{RESULTS}

\subsection{Morphological Observation of the 3D Outgrowths}

(Fig. 1) shows the evolution of a 3D human oral mucosa outgrowth during its first fifteen days in culture. Phase contrast microscopy (PhaCo) revealed that the 3D outgrowths initially formed a network of spindly cells (likely fibroblasts) and rounded cells (possibly epithelial cells), only visible at higher magnifications, growing out into the Matrigel ${ }^{\mathrm{TM}}$ from the biopsy which was origi-
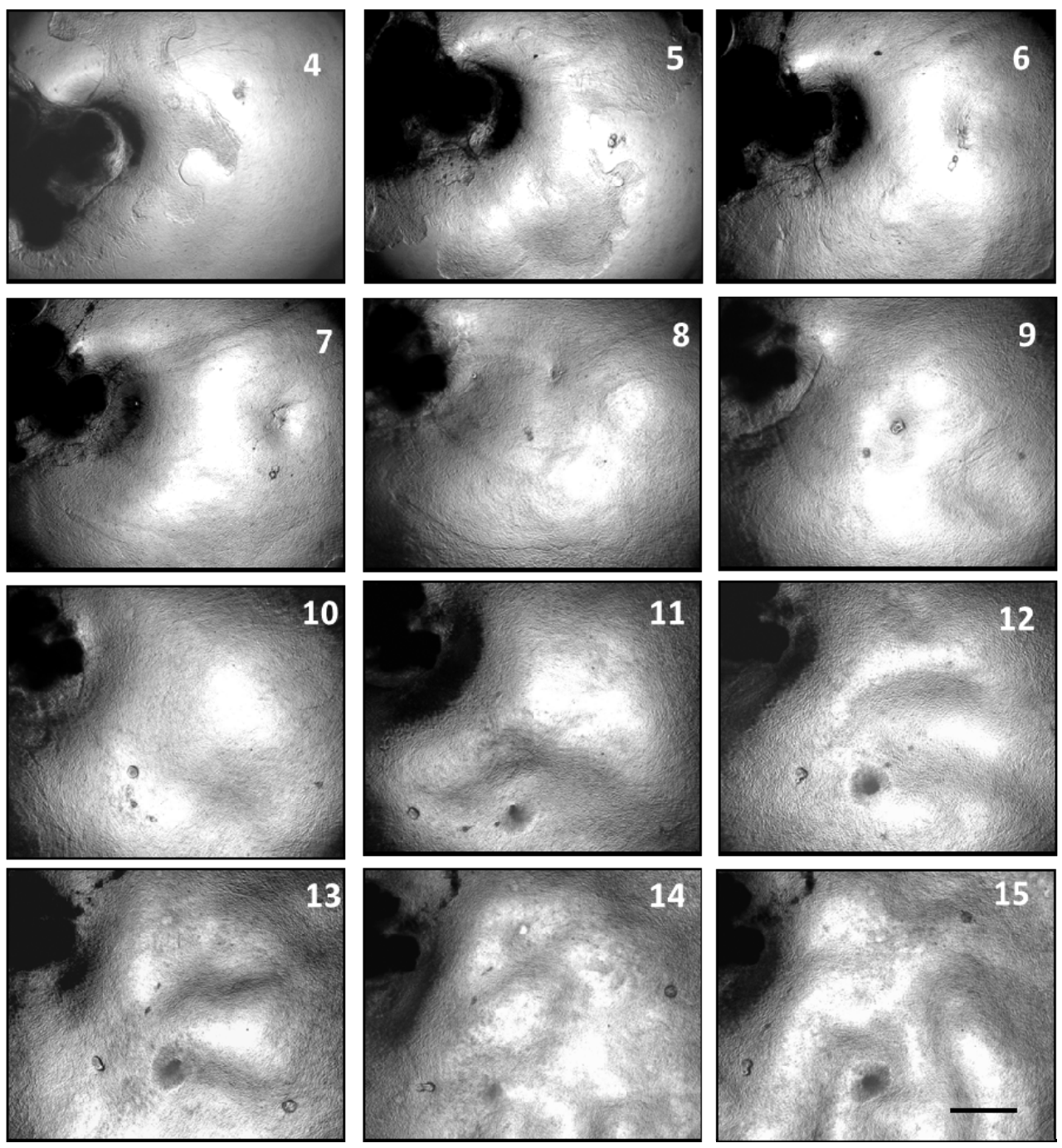

Fig. (1). Phase contrast observation of a 3D human oral mucosa outgrowth during its first 15 days in culture. During the first stages, mesenchymal cells emerge from the biopsy fragment, followed by epithelial cells. After around 7 days of culture, the cells have covered all the nylon membrane of the insert. Subsequently, the growth becomes three-dimensional. Bar $=2 \mathrm{~mm}$. 
nally placed in the middle of the transwell system (Fig. 1, day 5). After 7 days, the nylon membrane covering the bottom of the insert was completely covered with the newly grown tissue, and starting to develop three-dimensionally forming ridges and more complex 3D structures. After 13-15 days of culture, the morphological features of the outgrowths (as observed with the PhaCo) did not display any further significant changes. In our experience, unless specific damaging events (e.g. contamination with infectious organisms) occur during the culture period, and providing proper culture conditions are maintained, it is possible to continue to grow these $3 \mathrm{D}$ cultures for a period of up to two months.

\subsection{Transmission Electron Microscopy Characterization of the 3D Outgrowths}

In order to evaluate the ultra-structural features of our 3D human oral mucosa outgrowths, these were grown until differentiation was complete (around 14 days in most cases) and then prepared for TEM analysis as detailed in section 2.2.2.

(Fig 2) shows typical TEM micrographs obtained from a sample that was grown for 15 days. TEM analysis enabled us to identify in the outgrowths two different cell populations, non-keratinized epithelial cells above and fibroblasts underneath. The epithelial layer was composed of different strata, reminiscent of the basal, spinous, intermediate and superficial layers of the human oral mucosa in vivo. (Fig. 2A) is a low magnification view of the outgrowth that shows, in particular, the lowest three strata of the epithelial layer. The epithelial cells exhibit a squamous non-keratinized phenotype, typical of the oral lining mucosa, and as better illustrated with a higher magnification in (Fig. 2B), epithelial interactions are guaranteed by tight junction devices (desmosomes, white arrows). (Fig. 2C) shows a particular of the fibroblast layer (lamina propria) that displays the characteristic spindly morphology of these cells and also the presence of an abundant extracellular matrix; note that it was not possible to discriminate between the papillary and reticular layer in this region, suggesting that mechanical forces, clearly not present in this model, could be responsible for the development of these two layers in vivo. (Fig. 3A) shows a highly magnified region of the interface between the basal and spinous layers, with the two adjacent cytoplasmic membranes of two keratinocytes tightly held together by a desmosome (white arrows) with both the attachment plaques well evident, together with the intermediate filaments starting from them. In this region, it is also interesting to note tightly packed cytokeratin filaments (tonofibrils, white arrowheads) interacting with the desmosomal structure; this is a typical morphological feature of the cytoskeleton of the basal keratinocytes of human oral mucosa. In (Fig. 3B) it is possible to observe that the basal epithelial cells are separated from the underlying fibroblast layer by a properly constituted basal membrane with which they both interact through hemidesmosomes (white arrows); in this micrograph it is only possible to observe the two most superficial regions of the basal membrane, the lamina lucida and densa, because the innermost one, the lamina reticularis, is lost during the fixation procedure that unfortunately determines a detachment of the epithelial layers from the fibroblastic one. (Fig. 3C) shows details of a cytoplasmic process of a fibroblast: it is quite clear that the proteic material (white arrows) that will eventually form the ECM is extruded into the extracellular space from caveolae-like structures (white arrowhead) present on the cytoplasmic membrane of the fibroblasts. This proteic material is mainly constituted by collagen proteins, as shown in (Fig. 3D). cular.

Both the epithelial and fibroblast layers in this model are avas-

Moreover, it was not possible to identify any other cytotypes (such as immune cells or melanocytes) apart from the ones already described. This characteristic is another benefit of our model, because it enables the study of cellular processes devoid of the presence of inflammatory cells and their products.
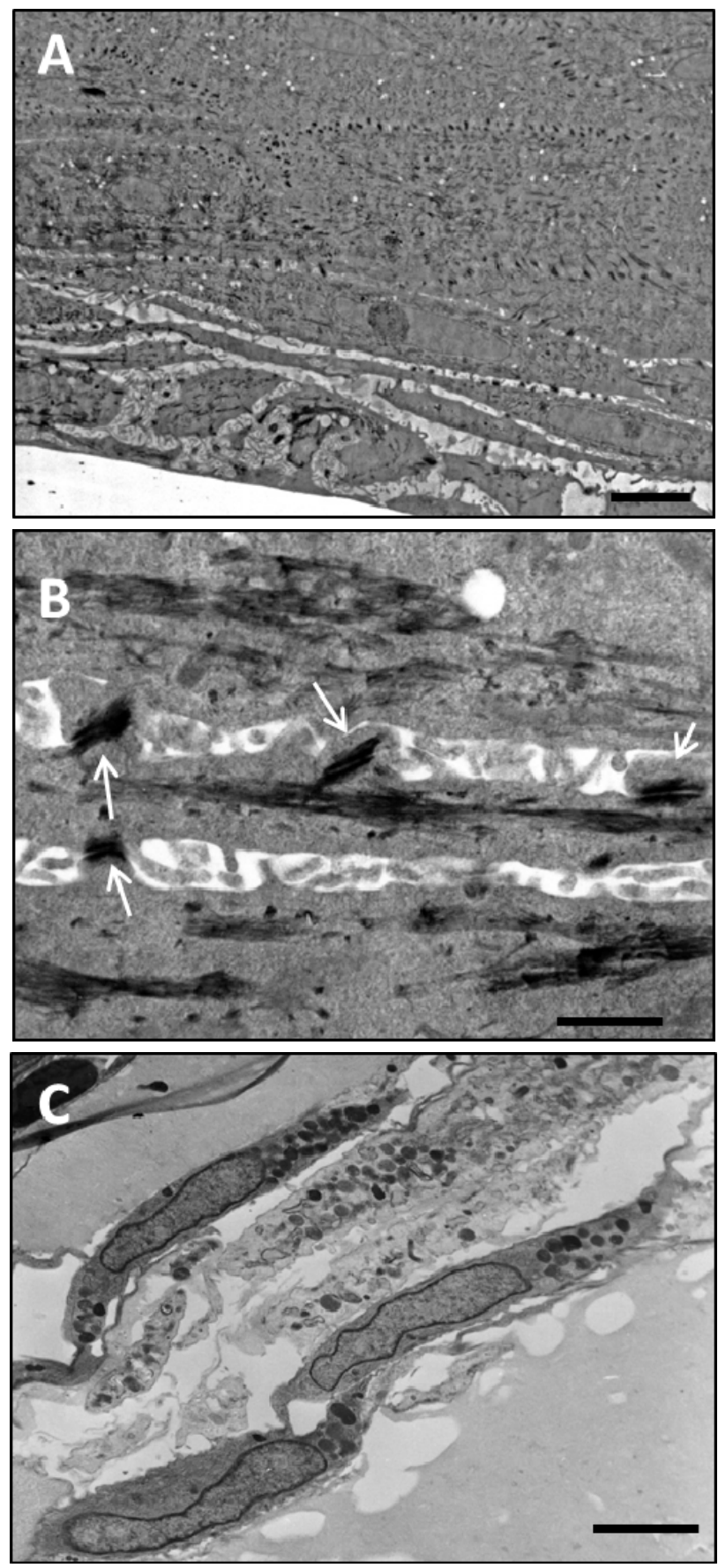

Fig. (2). TEM observation of a 3D outgrowth after 15 days in culture. In (A) is shown the epithelial layer with its basal, spinous and intermediate layers. (B) is a higher magnification of the interface between the basal and spinous layers of the epithelial layer. In $(\mathbf{C})$ is shown the fibroblast layer (lamina propria) where fibroblasts are surrounded by ECM proteins. Bars in A and $\mathrm{C}=10 \mu \mathrm{m}$, bar in $\mathrm{B}=400 \mathrm{~nm}$

\subsection{Immunophenotipical Characterization}

\subsubsection{Immunofluorescence}

In order to properly characterize our 3D outgrowths, these were stained with a panel of antibodies directed towards some of the most common markers of the human oral mucosa components, and visualized by laser confocal microscopy in order to perform precise scans at the desired level of the different strata composing the 

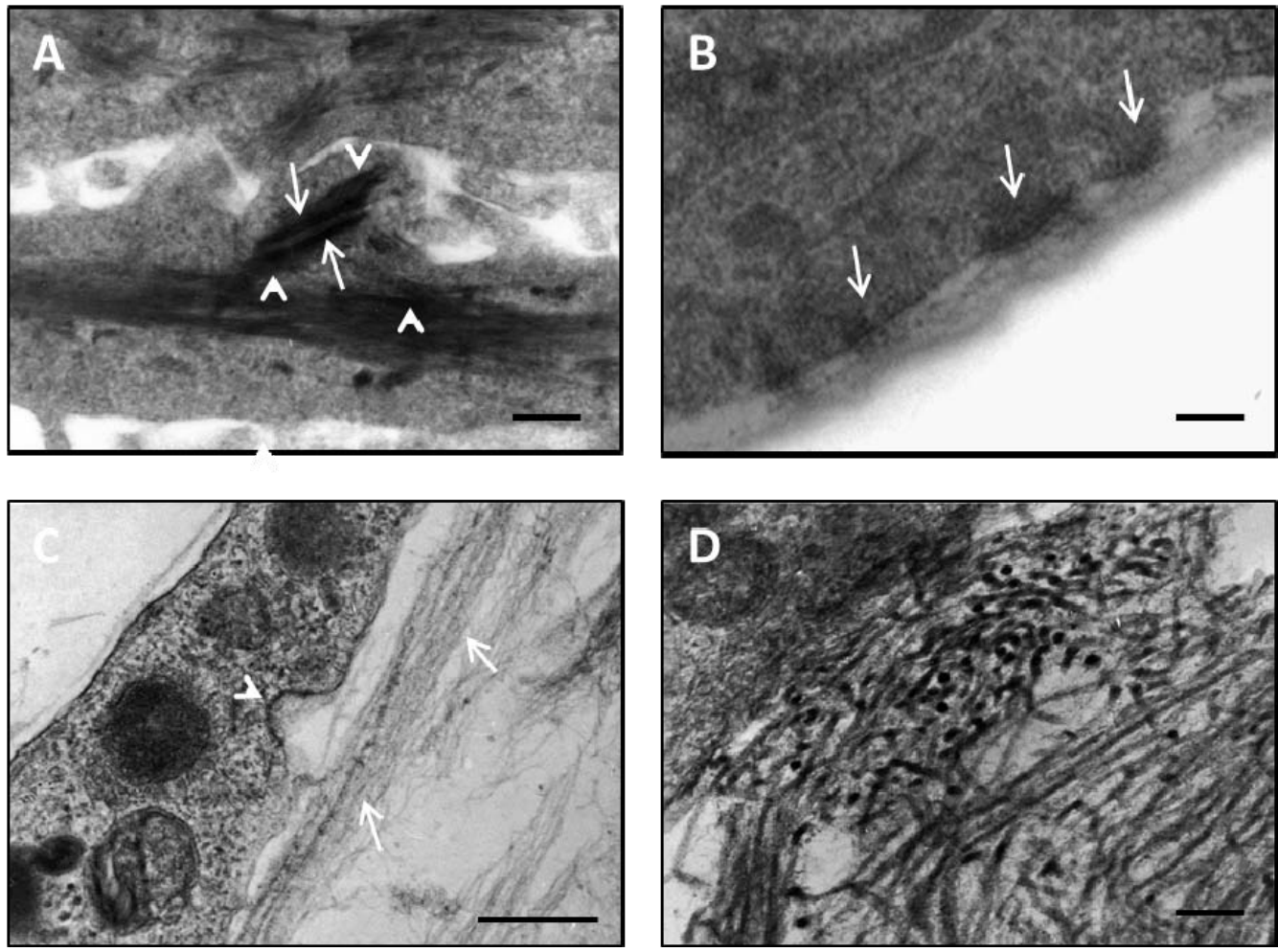

Fig. (3). High magnification TEM analysis of keratinocytes and fibroblasts in a 15 days old 3D outgrowth. (A) shows a highly magnified region of the interface between the basal and spinous layers, displaying the two adjacent cytoplasmic membranes of two keratinocytes held tightly together by a desmosome (white arrows) from which tonofibrils (white arrowheads) project into the cytoplasm; (B) shows the basal epithelial cells, separated from the underlying fibroblasts layer by a properly constituted basal membrane with which they both interact through hemidesmosomes (white arrows); (C) shows details of a fibroblast surrounded by ECM proteins. The cytoplasm of the fibroblasts contain vacuoles filled with proteic material and their cytoplasmic membranes present caveolae-like structures (white arrowhead), from where the assembled proteins (white arrows) are released into the extracellular space. This proteic material is mainly constituted by collagen proteins, as shown in $(\mathbf{D})$. Bars in $\mathbf{A}, \mathbf{B}$ and $\mathbf{C}=200 \mathrm{~nm}$, in $\mathbf{D}=50 \mathrm{~nm}$.

outgrowth. A complete list of all the antibodies employed can be found in Table 1.

(Fig. 4) shows four of the markers that were used to differentiate between the cell populations: CK5 and CK13 were employed to characterize basal and differentiated keratinocytes respectively (Fig. $\mathbf{4 A}$ and $\mathbf{B}$ ), whereas laminin (Fig. 4C) and collagen type IV (Fig 4D) were used, together with fibronectin and collagen type I (not shown), to study the ECM. CK5 staining was limited to the basal layer and positive cells had a distinctive rounded shape (Fig. 4A) whereas CK13 staining, that was present throughout the whole epithelial stratum, was much stronger in the upper layers and positive cells had a typical squamous shape (Fig. 4B).

Table 2 shows the scores obtained with our panel of antibodies in $3 \mathrm{D}$ outgrowths from six different subjects (\#1 to \#6). These data are compatible with a properly organized and differentiated normal oral mucosa. However, it is interesting to note that in our model Merkel cells (as identified by CK20), melanocytes (MTIF) and immune/inflammatory cells (CD3, CD4, CD8, CD45, CD68 and MPO) were completely absent.

\subsubsection{Immunogold.}

In order to verify whether the ECM proteins that were found in the lamina propria of our 3D outgrowths with the immunofluorescent staining, had effectively been produced by the resident fibroblasts, these proteins were precisely localized inside the fibroblasts cytoplasm by TEM immunogold assay. (Fig. 5) shows representative micrographs of immunogold staining of fibroblasts of the lamina propria of the 3D outgrowth, with antibodies directed towards laminin (Fig. 5A), fibronectin (Fig. 5B) and collagen type IV (Fig. 5C). All proteins analyzed were found to be expressed in the cyto- plasm of the fibroblasts, especially in the proximity of and within vacuolar cytoplasmic structures, with the exception of collagen type IV whose staining was diffuse.

\section{DISCUSSION}

Various cell-secreted macromolecular components make up the intricate, highly hydrated polymer gel that constitutes the threedimensional extracellular microenvironment. The ECM is composed of cross-linked, physically immobilized sugar and protein elements. Further components include growth factors, chemokines, cytokines and other soluble effectors with important signaling functions, as well as membrane-anchored molecules, donated by neighboring cells, that have the crucial task of enabling cell-cell communication in tissue morphogenesis. Fibrous proteins, such as elastin, fibronectin, laminins and collagens, and hydrophilic proteoglycans containing large glycosaminoglycan side chains, such as hyaluronic acid, are two of the principal ECM macromolecules. While these components are present in the majority of ECMs, their form and organization, as well as their biochemical and mechanical properties diverge considerably between different types of tissues. In addition to its basic functions as a solid support structure upon which cells are organized into 3D tissues, or simply as a physical boundary between neighboring tissues, ECM also regulates multiple morphogenesis-driving cellular processes, such as cell adhesion, migration, proliferation and division, via distinct receptor-ligand interactions $[14,15]$.

Notable progress has been made in the last decade or so in devising artificial ECMs. Suitable materials for productive use in three-dimensional cell cultures and in vivo tissue regeneration have 

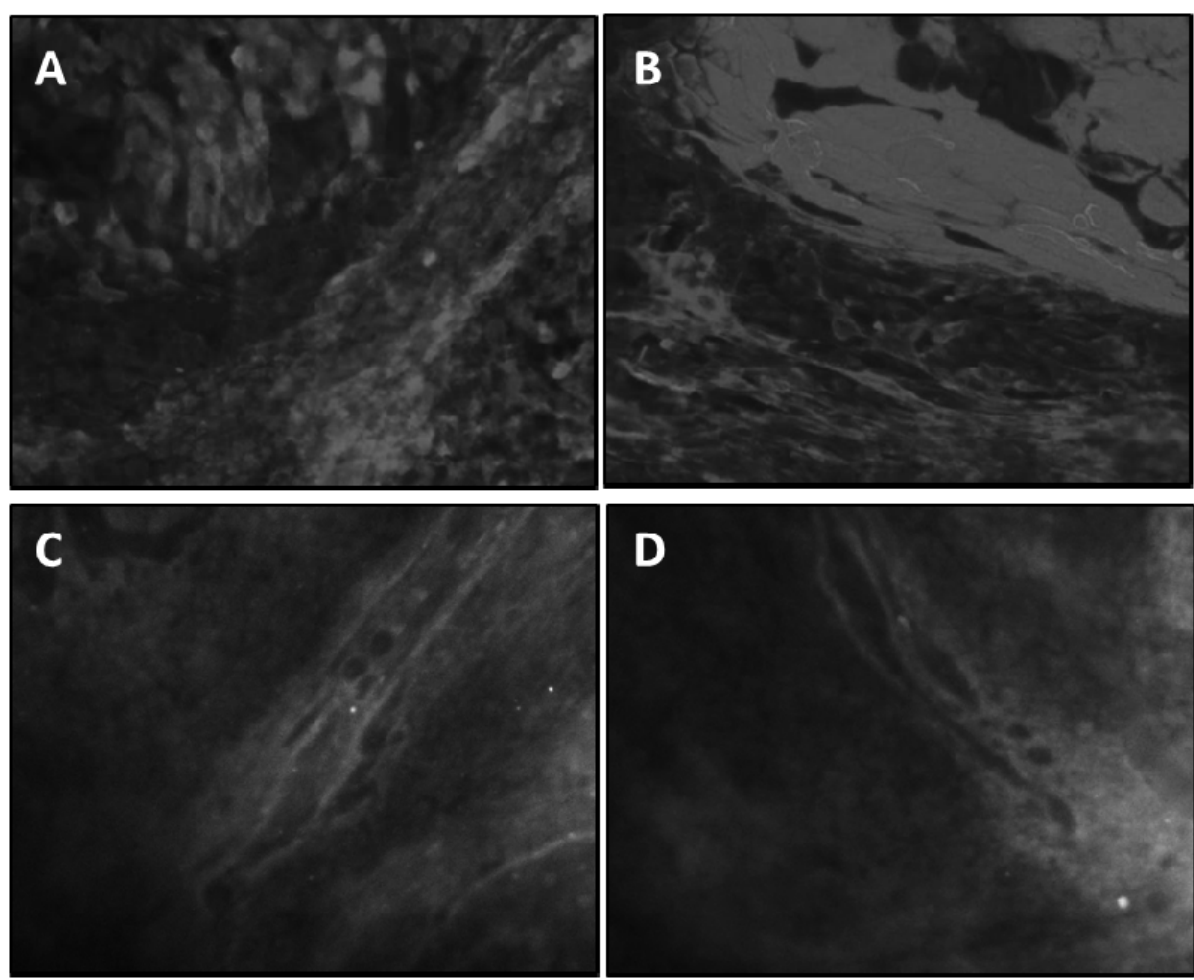

D

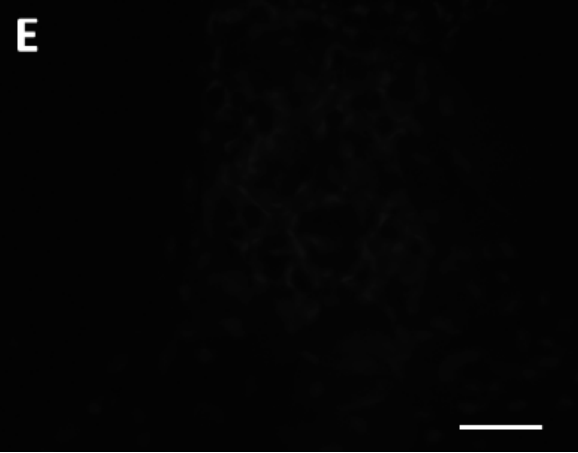

Fig. (4). Immunofluorescence staining of the epithelial (A and B) and fibroblast (C and D) layers in a 15 days old 3D outgrowth. The basal keratinocytes resulted positive for CK5 (A), whereas the more differentiated ones resulted positive for CK 13 (B); the fibroblast layer was positive for laminin (C) and collagen type IV (D). E shows a negative isotype control. Bar $=40 \mu \mathrm{m}$.

Table 2. Semi-quantification of the immunophenotypic analysis of the markers studied. 3D outgrowths from six different subjects were stained with several markers whose expressions were semi-quantified with the following scoring system: (-) absence of immunostaining, $(-/+)$ less than $10 \%$ of the total number of cells/area scored positively; (+) between $10 \%$ and $50 \%$ scored positively; $(++)$ $50-100 \%$ of the total number of cells/area were positive.

\begin{tabular}{|c|c|c|c|c|c|c|}
\hline & $\# 1$ & $\# 2$ & $\# \mathbf{3}$ & $\# 4$ & $\# 5$ & \#6 \\
\hline CK10 & - & - & - & $-/+$ & - & - \\
\hline CK13 & ++ & ++ & ++ & ++ & ++ & ++ \\
\hline CK20 & - & - & - & - & - & - \\
\hline Collagen I & ++ & ++ & ++ & ++ & ++ & + \\
\hline
\end{tabular}


(Table 2) Contd....

\begin{tabular}{|c|c|c|c|c|c|c|}
\hline & $\# 1$ & \#2 & \#3 & $\# 4$ & $\# 5$ & \#6 \\
\hline Collagen IV & + & + & + & + & + & + \\
\hline Laminin & + & + & + & $-1+$ & + & + \\
\hline Fibronectin & + & + & ++ & + & + & + \\
\hline E-cadherin & ++ & ++ & ++ & + & ++ & ++ \\
\hline Involucrin & + & $-/+$ & - & $-1+$ & - & $-1+$ \\
\hline MITF & - & - & - & - & - & - \\
\hline CD3 & - & - & - & - & - & - \\
\hline CD4 & - & - & - & - & - & - \\
\hline CD8 & - & - & - & - & - & - \\
\hline CD45 & - & - & - & - & - & - \\
\hline CD68 & $-1+$ & - & - & $-1+$ & - & - \\
\hline MPO & - & - & - & - & $-/+$ & - \\
\hline
\end{tabular}
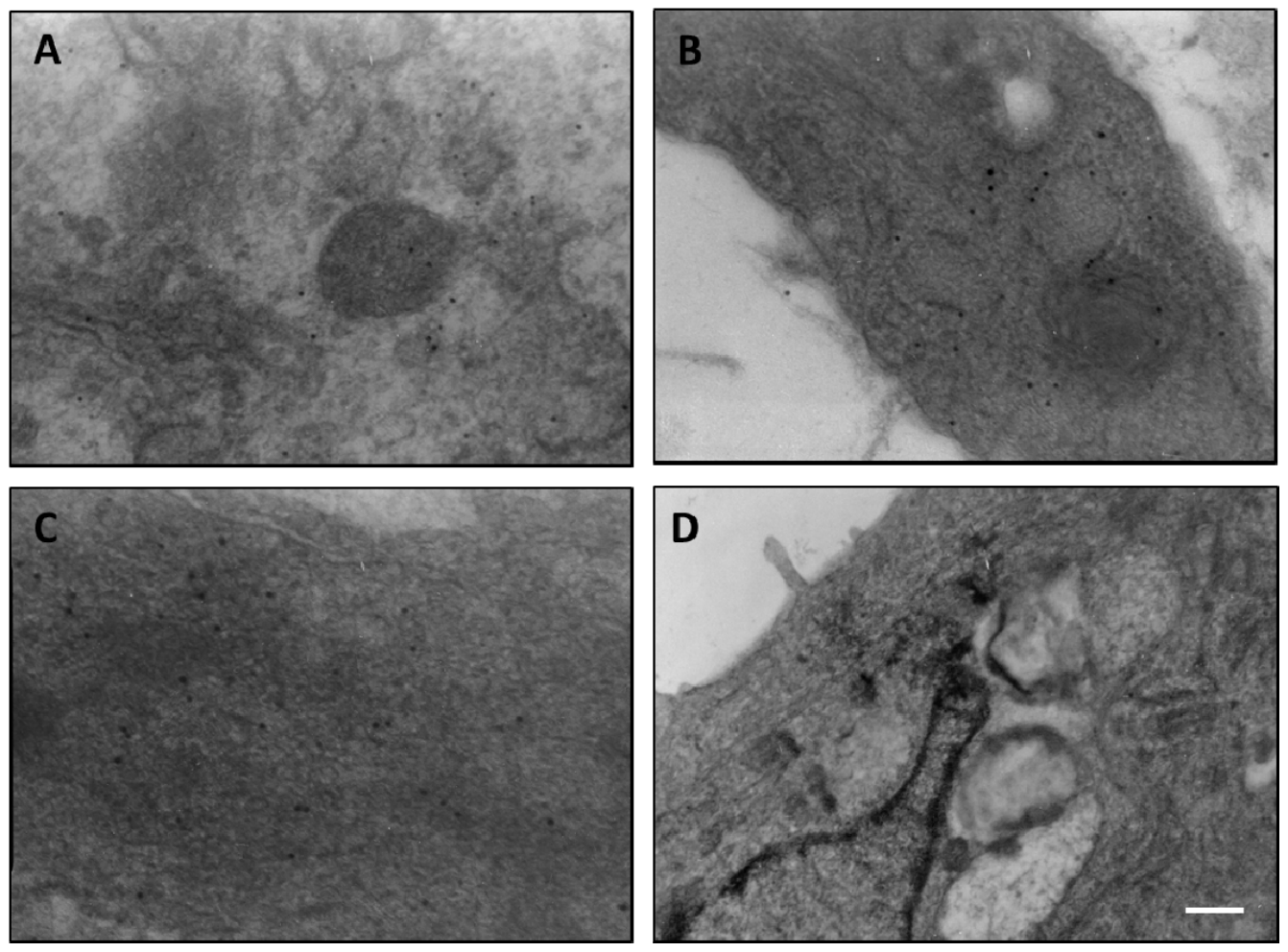

Fig. (5). Immunogold staining of fibroblast cytoplasmic proteins. A 15 days old 3D outgrowth of human oral mucosa was immunostained for laminin (A), fibronectin (B) and collagen type IV (C) using secondary antibodies labelled with colloidal gold particles 10nm in diameter and then analysed by TEM. D shows a negative isotype control. $\mathrm{Bar}=50 \mathrm{~nm}$.

become a reality thanks to the application of biological recognition principles. Nonetheless, these artificial systems can still only reiterate a small portion of the key signaling and cell response functions of natural ECMs, with a few characteristic functions remaining beyond the grasp of the synthetic versions at the moment. These include the near-physiological multifunctional capabilities of the ECM; while most artificial ECMs utilize two classes of biomolecules, the natural ones are comprised of various different bio- chemical cues. Additionally, artificial ECMs lack in the temporal complexity in signal presentation, with the time frames and dynamics being fairly restricted in terms of longevity and complexity. Spatial complexity represents a further shortcoming of artificial ECMs. Matrix-immobilized 3D morphogen gradients are an essential factor in tissue development and regeneration, enabling several cell types and patterns to be spatially generated in relation to the original signal source, as well as controlling the migration of spe- 
cific cells to specific locations. Although biomolecule gradients have been created using hydrogel surfaces $[16,17]$, to date no one has succeeded in recreating them in artificial cell-responsive 3D ECMs. The versatility of artificial ECMs is also hindered by the absence of a suitable feedback system to control cell-matrix interactions. Characteristic of natural ECMs, these include features such as proteases capable of cleaving ECM components, generating cleavage products that can have important signaling functions [18]. Moreover, most artificial ECMs currently available have some cellspecificity issues, since protease substrates of cell-responsive matrices consist of short, linear peptides with limited specificity for particular proteases; this is not the case in matrices composed of natural proteins. Therefore, most artificial ECMs are not specific to particular cells or their protease secretions, respectively. Another important factor that must be considered in oral mucosa model reconstruction is the type and origin of fibroblasts and keratinocytes. Fibroblasts are usually isolated from the dermal layer of the skin or obtained by oral mucosal biopsy, and used for tissue engineering at early passages, because their ECM production decreases as the passage number increases [1]. Keratinocytes can be obtained from different sites of the oral cavity, such as the hard palate [9], gingiva [10] or buccal mucosa [11]. Normal human keratinocytes should also be used at very early passages, but immortalized human keratinocytes, such as HaCaT cells [12] or TR146 cells [13], can be used at extended passages in the reconstruction of oral mucosal test models. However, epidermal differentiation of transformed keratinocytes is not perfect, and tumor derived cells are abnormal and not suitable for clinical use [1]. The results that we obtained during the development and characterization of our 3D model of the human oral mucosa clearly show that this system is a very good candidate for tissue-engineering the normal human oral mucosa. In our model, oral biopsies are placed directly on Matrigel ${ }^{\mathrm{TM}}$ in Transwells equipped with a nylon membrane necessary to allow the passage of culture medium. Cells from our 3D outgrowth find in the Matrigel $^{\mathrm{TM}}$ all the factors necessary to develop and to reconstitute the structures of the original tissue. However, after this initial phase, cells within the outgrowth start to demolish the Matrigel ${ }^{\mathrm{TM}}$ and begin to lay down their own ECM. This is a crucial moment in the development of the 3D outgrowth, resulting in a proper differentiation of the cell components (keratinocytes and fibroblasts) and in an in vitro reconstitution of the physiological architecture of human oral mucosa, including a stratified non-keratinized squamous layer composed of several layers, a proper basal membrane and a lamina propria where fibroblasts continue to produce ECM and biochemically signal with the layers above.

Melanocytes, Merkel cells and immune cells are not present in our 3D outgrowth model. As stated earlier, this is not necessarily a negative aspect, as it in fact enables us to study the eventual responses of the oral mucosa to exogenous molecules (such as drugs, growth factors, cytokines etc.) in the absence of confounding factors and signals produced by the immune cells, thus rendering this model a valid tool for studying, for example, the effectiveness of drugs administration.

Even if Matrigel ${ }^{\mathrm{TM}}$ is considered one of the most effective substrates for 3D cell culture, we believe that the strength of our outgrowth model is not so much in the culture medium used or in the matrix, but in the common source of fibroblasts and keratinocytes. In fact, fibroblasts and keratinocytes of our model are not separately isolated from the dermal layer or buccal mucosa respectively, and combined again in a scaffold of different materials, but they originate from the same biopsy. In this manner, the cultured tissue maintains the distinct characteristics of the single original source.

\section{CONCLUSIONS}

Primary cultures or cell lines still represent the most commonly used human in vitro culture models to study responses of cell systems to specific stimuli. However, the main limitations of these models are the absence of extracellular components and the loss of proper cell-cell communication that arises from the former characteristic. In our model, both keratinocytes and fibroblasts outgrow autonomously from an oral biopsy into a 3D gel (Matrigel ${ }^{\mathrm{TM}}$ ), whose composition is very similar to that of the normal ECM of the oral mucosa. Moreover, after the initial expansion phase, fibroblasts start to lay out a newly formed ECM that is architecturally and structurally compatible with that of the in vivo human oral mucosa. In the meantime, the keratinocytes start differentiating because of the air-liquid interface, and after around 15 days of culture the outgrowths present a properly differentiated oral epithelium separated, by a functional basal membrane, from a newly constituted lamina propria where fibroblasts lay the ECM. In our opinion, this constitutes a valid model where to study the responses of the human oral mucosa as a whole, to the administration of drugs and other exogenous substances.

\section{CONFLICT OF INTEREST}

The authors confirm that this article content has no conflicts of interest.

\section{ACKNOWLEDGEMENT}

This research was partly funded by the grant "Progetti Innovativi D'Ateneo - 2007" of the University of Palermo.

\section{REFERENCES}

[1] Moharamzadeh K, Brook IM, Van Noort R, Scutt AM, Thornhill MH. Tissue-engineered oral mucosa: a review of the scientific literature. J Dent Res 2007; 86(2): 115-24.

[2] Kinikoglu B, Rodríguez-Cabello JC, Damour O, Hasirci V. The influence of elastin-like recombinant polymer on the self-renewing potential of a 3D tissue equivalent derived from human lamina propria fibroblasts and oral epithelial cells. Biomaterials 2011; 32(25): 5756-64.

[3] Rheinwald JG, Green H. Serial cultivation of strains of human epidermal keratinocytes: the formation of keratinizing colonies from single cells. Cell 1975; 6: 331-43.

[4] Moharamzadeh K, Brook IM, Van Noort R, Scutt AM, Smith KG, Thornhill MH. Development, optimization and characterization of a full-thickness tissue engineered human oral mucosal model for biological assessment of dental biomaterials. J Mater Sci Mater Med 2008; 19(4): 1793-801.

[5] Hodde J. Naturally occurring scaffolds for soft tissue repair and regeneration. Tissue Eng. 2002; 8(2): 295-308.

[6] Gentzkow GD, Iwasaki SD, Hershon KS, et al. Use of dermagraft, a cultured human dermis, to treat diabetic foot ulcers. Diabetes Care 1996; 19: 350-4.

[7] Moriyama T, Asahina I, Ishii M, Oda M, Ishii Y, Enomoto S. Development of composite cultured oral mucosa utilizing collagen sponge matrix and contracted collagen gel: a preliminary study for clinical applications. Tissue Eng 2001; 7: 415-27.

[8] Kim J, Suh SW, Shin JY, Kim JH, Choi YS, Kim H. Replacement of a tracheal defect with a tissue-engineered prosthesis: early results from animal experiments. J Thorac Cardiovasc Surg 2004; 128: $124-9$.

[9] Cho KH, Ahn HT, Park KC, et al. Reconstruction of human hardpalate mucosal epithelium on deepidermized dermis. J Dermatol Sci 2000; 22: 117-24.

[10] Yoshizawa M, Feinberg SE, Marcelo CL, Elner VM. Ex vivo produced human conjunctiva and oral mucosa equivalents grown in a serum-free culture system. J Oral Maxillofac Surg 2004; 62: 980-8.

[11] Bhargava S, Chapple CR, Bullock AJ, Layton C, MacNeil S. Tissue engineered buccal mucosa for substitution urethroplasty. BJU Int 2004; 93: 807-11.

[12] Boelsma E, Verhoeven MC, Ponec M. Reconstruction of a human skin equivalent using a spontaneously transformed keratinocyte cell line (HaCaT). J Invest Dermatol 1999; 112: 489-98.

[13] Schmalz G, Schweikl H, Hiller KA. Release of prostaglandin E2, IL-6 and IL-8 from human oral epithelial culture models after exposure to compounds of dental materials. Eur J Oral Sci 2000; 108: 442-8. 
[14] Giancotti FG, Ruoslahti E. Integrin signaling. Science1999; 285(5430): 102832 .

[15] Kleinman HK, Philp D, Hoffman MP. Role of the extracellular matrix in morphogenesis. Curr Opin Biotechnol 2003; 14(5): 52632.

[16] Burdick JA, Khademhosseini A, Langer R. Fabrication of gradient hydrogels using a microfluidics/photopolymerization process. Langmuir 2004; 20(13): 5153-6.
[17]

DeLong SA, Moon JJ, West JL. Covalently immobilized gradients of bFGF on hydrogel scaffolds for directed cell migration. Biomaterials 2005; 26(16): 3227-34.

[18] Hamano Y, Zeisberg M, Sugimoto H, et al. Physiological levels of tumstatin, a fragment of collagen IV alpha 3 chain, are generated by MMP-9 proteolysis and suppress angiogenesis via alpha $\mathrm{V}$ beta 3 integrin. Cancer Cell 2003; 3(6): 589-601.

[19] Hughes CS, Postovit LM, Lajoie GA. Matrigel: a complex protein mixture required for optimal growth of cell culture. Proteonomics 2010; 10: 1886-90. 